Rev. salud pública. 14 (2): 296-304, 2012

\title{
Prevalencia de helmintos en la planta de aguas residuales del municipio EI Rosal, Cundinamarca
}

\author{
Helminth prevalence in a waste-water plant at El Rosal, \\ Cundinamarca
}

\author{
Carolina Ortiz, Myriam C. López y Favio A. Rivas \\ Departamento de Salud Pública, Facultad de Medicina. Universidad Nacional de Colombia. Bogotá, \\ Colombia.cortizpi@unal.edu.co; mclopezp@unal.edu.co; farivasm@unal.edu.co
}

Recibido 13 Septiembre 2011/Enviado para Modificación 5 Febrero 2012/Aceptado 5 Abril 2012

\section{RESUMEN}

Objetivo Determinar la prevalencia de huevos de helmintos en lodos, agua residual cruda y tratada, provenientes de un sistema de tratamiento de aguas residuales del municipio el Rosal, Cundinamarca.

Metodología Se tomaron 30 muestras de agua residual, y 10 de lodos en la Planta El Rosal, durante 10 semanas. Las muestras de aguas y lodos se procesaron siguiendo los métodos de BAILENGER, y el método de la Norma Oficial Mexicana, respectivamente. La viabilidad de los huevos se determinó por el método de Victórica \& Galván y la Norma Oficial Mexicana.

Resultados Los datos fueron analizados con estadística descriptiva. El 100 $\%$ de las muestras de agua residual sin tratar evidenció presencia de huevos, encontrándose al menos un huevo viable de helminto/litro en el $90 \%$ de las mismas. El $90 \%$ de las aguas residuales tratadas fueron positivas para la presencia de huevos, encontrándose que el $70 \%$ presentaba al menos 1 huevo viable. Todas las muestras de agua residual cruda que se vierten directamente a la quebrada, fueron positivas a helmintos, igual situación se encontró al momento de realizar la prueba de viabilidad. Todos los lodos fueron positivos para helmintos, encontrándose que en el $100 \%$ de estos, al menos un huevo fue viable.

Conclusión El uso de estas aguas para riego de hortalizas, y el uso de estos lodos como abono, representa un riesgo potencial para la salud pública. Los lodos solo pueden ser usados en actividades forestales, siempre y cuando no estén en contacto con humanos.

Palabras Clave: Epidemiologia, helmintiasis, aguas residuales, salud pública (fuente: DeCS, BIREME). 


\section{ABSTRACT}

Objective Assessing helminth egg prevalence in sludge and raw and treated wastewater from a wastewater treatment system located in the village of El Rosal, Cundinamarca.

Methodology 30 wastewater and 10 sludge samples from the El Rosal plant were taken during a 10-week period. The sludge and water samples were processed according to the Bailinger and the official Mexican standard methodology, respectively. Egg viability was determined by the method described by Victórica \& Galván and the Mexican official standard.

Results Descriptive statistics were used for analysing data. $100 \%$ of the untreated wastewater samples showed the presence of eggs and at least one viable helminth egg/litre was found in $90 \%$ of them. $90 \%$ of the treated wastewater samples were positive for the presence of eggs, finding that $70 \%$ had at least one viable egg. All raw wastewater samples being dumped directly into the stream were positive for helminths; the same situation was found at the time of the viability test. All sludge samples were positive for helminths, finding that $100 \%$ of these had at least one viable egg.

Conclusion Using this water for crop irrigation and using the sludge as fertiliser is a potential risk for public health. The sludge can only be used in forestry activities, as long as it does not come into contact with humans.

Key Words: Epidemiology, helminthiasis, sewage, public health (source: $\mathrm{MeSH}$, $N L M)$.

$\mathrm{E}$ n el mundo, 2000 millones de personas están infectadas con parásitos intestinales, principalmente en países en vías de desarrollo (1). Estas enfermedades causan unas 9400 defunciones cada año (2). En Colombia, la encuesta de parasitismo intestinal, evidenció que el $81,1 \%$ de las personas se encontraban parasitadas (3). La asociación con contaminación fecal del suelo y de los alimentos, la insuficiencia de agua potable, la utilización de las aguas residuales, ausencia de saneamiento ambiental y condiciones socioeconómicas vulnerables, contribuyen a que continúen siendo un problema de salud pública en el país (4).

El crecimiento desmedido de la población, ha incrementado la demanda del recurso hídrico y a su vez, los vertimientos de aguas residuales domésticas han ido en aumento, por lo tanto, los niveles de contaminación han superado la capacidad de autodepuración de los cuerpos de agua. La Organización Mundial de la salud (OMS) reconoce los beneficios del uso de las aguas residuales adecuadamente tratadas, para el riego de cultivos y buscan fomentar la utilización segura de estas. Las aguas residuales contienen gran variedad y diversidad de microorganismos, entre ellos 
helmintos que pueden causar enfermedades al llegar a través del agua (5). La práctica de regar hortalizas con aguas sin tratar, o abonar los cultivos con lodos o biosólidos sin tratamiento previo, ha conducido a una pérdida de calidad de los productos con la consecuente exclusión de mercados a nivel regional, nacional e internacional.

En Colombia, se han realizado estudios sobre el desempeño del tratamiento de las aguas residuales y la aplicación de biosólidos en la agricultura (6). Sin embargo, son muy pocos los datos que se tienen sobre la viabilidad de los huevos de helmintos, siendo éste el parámetro de mayor importancia a evaluar (7).

En algunos municipios de la sabana de Bogotá que poseen plantas de tratamiento de aguas residuales, el agua de sus efluentes no se recomienda para uso agrícola por su alto contenido de coliformes totales. Sumado a esto, se desconoce la prevalencia de huevos de helmintos, que son sin duda, el mejor indicador de su calidad microbiológica. Este trabajo estima la prevalencia de huevos de helmintos en lodos, aguas residuales con y sin tratamiento provenientes del Municipio El Rosal, Cundinamarca, durante el año 2009. La planta de tratamiento fue elegida para este estudio teniendo en cuenta los siguientes criterios de inclusión: sus aguas son vertidas directamente a un afluente del rio Subachoque, estas aguas son usadas para el riego de cosechas. Con estos resultados se busca proponer soluciones que garanticen y minimicen el impacto que pueda causar la presencia de este tipo de microorganismos en la población que use el recurso hídrico.

\section{METODOLOGÍA}

Este trabajo se realizó en la planta de tratamiento de aguas residuales (PTAR) del municipio El Rosal, ubicado aproximadamente a 22 kilómetros de Bogotá. El sistema comprende un tratamiento primario de cribado y desarenado y uno secundario donde se utiliza un zanjón de oxidación y un sedimentador. Los lodos que no se recirculan, pasan a los lechos de secado, los cuales posteriormente son removidos hacia su sitio de disposición final.

\section{Muestras}

Se evaluaron aguas residuales crudas y tratadas, lodos de cámaras y de lechos de secado. Se muestreó durante los meses de junio a agosto de 2009, en el afluente y efluente, las muestras de agua se recogieron una vez por 
semana, para un total de 30 muestras, distribuidas de la siguiente manera: 10 de aguas residuales crudas, 10 de aguas residuales tratadas y 10 de agua residual cruda que se vertía directamente a la quebrada. Las muestras de lodos se tomaron cada 15 días, para un total de 10 muestras, donde 5 corresponden a lodos provenientes de la cámara y 5 a lodos del lecho de secado. Las muestras se analizaron en el laboratorio de parasitología de la facultad de medicina de la Universidad Nacional de Colombia, utilizando el método de Bailenger (9), y el método de la Norma Oficial Mexicana (10). La viabilidad de los huevos se determinó por la técnica de Victórica \& Galván (11), y el método de incubación.

Análisis Estadístico

A los resultados obtenidos se les realizó un análisis descriptivo, para determinar prevalencia, promedio y rango, de huevos de helmintos en muestras de lodos, agua residual cruda y tratada.

Se realizó una comparación entre la cantidad y la viabilidad de los huevos de helmintos que entraron a la planta de tratamiento de aguas residuales del municipio El Rosal y los que salieron, permitiendo establecer conclusiones acerca de la eficiencia de este sistema en la remoción de este tipo de patógenos.

\section{RESULTADOS}

Se evidenció que el 97,5\% de las aguas residuales, fueron positivas con algún huevo de helminto, siendo Áscaris sp el parásito más predominante, en un $92,5 \%$ de las muestras y un promedio de 51,3 huevos por litro (h/L), Uncinaria sp (35\%) con un promedio de 8,4 h/L, Hymenolepis diminuta ( $20 \%$ ) con un promedio de 7,2 h/L, Tricocéfalo $(12,5 \%$ ) con un promedio de 7,9 h/L, Hymenolepis nana (7,5 \%) con un promedio de 1,2 $\mathrm{h} / \mathrm{L}$. En la viabilidad por colorantes, al menos 1 huevo viable de Áscaris sp, estuvo presente en el 76,6 \% de las muestras, seguido por Tricocéfalo e Hymenolepis diminuta, en un 3,3 \%. Para la viabilidad por incubación, se observa que al menos 1 huevo viable de Áscaris sp, estuvo presente en el 43,3\%, seguido por Hymenolepis diminuta (6,7\%). La prevalencia de helmintos en las muestras de agua se observan en la Tabla 1. 
Tabla 1. Huevos de helmintos observados en las

\begin{tabular}{lcccccc}
\hline \multicolumn{2}{c}{ muestras de agua Residual } \\
\hline Especie Parasitaria & $\begin{array}{c}\text { Agua residual } \\
\text { sin tratamiento }\end{array}$ & $\begin{array}{c}\text { Agua residual sin } \\
\text { tratamiento que se } \\
\text { vierte directamente } \\
\text { a la Quebrada }\end{array}$ & $\begin{array}{c}\text { Agua residual } \\
\text { tratada }\end{array}$ \\
\cline { 2 - 6 } & $\begin{array}{c}\text { Promedio } \\
\text { huevos/litro }\end{array}$ & $\%$ & $\begin{array}{c}\text { Promedio } \\
\text { huevos/litro }\end{array}$ & $\%$ & $\begin{array}{c}\text { Promedio } \\
\text { huevos/litro }\end{array}$ & $\%$ \\
\hline Áscaris sp & 72 & 90 & 79 & 100 & 2,8 & 80 \\
Uncinaria sp & 12,1 & 60 & 12,3 & 50 & 0,8 & 30 \\
Trichuris trichiura & 1,6 & 1,6 & 5 & 20 & 0,7 & 20 \\
Hymenolepis diminuta & 8,1 & 40 & 13,3 & 40 & 0 & 0 \\
Hymenolepis nana & 1,8 & 20 & 1,6 & 10 & 0 & 0 \\
\hline
\end{tabular}

Para los lodos, se observó que el $100 \%$ de las muestras fueron positivas para huevos de Áscaris sp, con un promedio de 33,5 huevos en 2 gramos de sólidos totales ( $2 \mathrm{~g} / \mathrm{ST})$, Hymenolepis diminuta (100) con un promedio de 4,5 huevos en $2 \mathrm{~g} / \mathrm{ST}$. Tricocéfalo $(90 \%)$ con un promedio de 3,5 huevos en $2 \mathrm{~g} / \mathrm{ST}$, Uncinaria sp (90\%) con un promedio de 2,5 huevos en $2 \mathrm{~g} / \mathrm{ST}$, Toxocara sp $(50 \%)$ con un promedio de 1 huevo en $2 \mathrm{~g} / \mathrm{ST}$, Taenia sp (20\%) con un promedio de 0,2 huevos en $2 \mathrm{~g} / \mathrm{ST}$. En la prueba de vialidad por colorantes, al menos 1 huevo viable de Áscaris sp, estuvo presente en el $100 \%$ de las muestras, y en la viabilidad por incubación, al menos 1 huevo viable de Áscaris sp, estuvo presente en el $100 \%$ de las muestras. La prevalencia de helmintos en las muestras de lodos se observa en la Tabla 2.

Tabla 2. Huevos de helmintos observados en las muestras de lodos

\begin{tabular}{lcccc}
\hline \multirow{2}{*}{ Especie Parasitaria } & \multicolumn{2}{c}{ Cámara de Lodos } & \multicolumn{2}{c}{ Lodos lechos de secado } \\
\cline { 2 - 5 } & Promedio huevos/litro & $\%$ & Promedio huevos/litro & $\%$ \\
\hline Ascaris $\mathrm{sp}$ & 27 & 100 & 40 & 100 \\
Uncinaria $\mathrm{sp}$ & 1,4 & 80 & 3,6 & 100 \\
Trichuris trichiura & 1,8 & 80 & 5,2 & 100 \\
Hymenolepis diminuta & 5 & 100 & 4 & 100 \\
Toxocara sp & 1 & 60 & 1 & 40 \\
Taenia sp & 0.2 & 20 & 0,2 & 20 \\
\hline
\end{tabular}

\section{DISCUSIÓN}

La PTAR del municipio El Rosal, se diseñó con capacidad de 26.39 litros/ segundo, pero esta es insuficiente ya que no puede tratar la totalidad de las aguas residuales que se generan en el municipio, por lo tanto sólo se le realiza tratamiento a una parte de las aguas que llegan a este sistema y la otra se vierte directamente sin ningún tipo de tratamiento a la quebrada El Rosal, dato que concuerda con lo informado por Salas (12) y Silva (13), 
donde mencionan que solo el $8 \%$ del total de las aguas residuales que se producen en Colombia es tratado.

Se observó que los lodos que se generan del tratamiento de las aguas residuales, no son dispuestos adecuadamente, ya que una vez finalizado su ciclo de secado, son puestos en la parte posterior de la planta de tratamiento, a orillas de la quebrada El Rosal, donde por escorrentía van cayendo poco a poco al cuerpo de agua. Este factor de contaminación es muy importante a tener en cuenta, ya que los lodos concentran gran cantidad de huevos de helmintos, y debido a sus características físico-químicas, deben ser sometidos a tratamientos adecuados tales como, estabilización alcalina con altas temperaturas. La remoción de huevos de helmintos debe ser efectiva, ya que los lodos, son ambientes propicios para el embrionamiento de los huevos que se encuentren viables, como lo describe Maya (14) y Jiménez (15).

Es lógico suponer la presencia de estos huevos en cantidades considerables en las aguas residuales, resultados que contrastan por lo analizado por Campos (16), quien utilizó el procedimiento recomendado por la Norma Oficial Mexicana, encontrando que la concentración de huevos de helmintos en la planta de aguas residuales El Salitre de Bogotá y en los canales de riego del distrito de la ramada, es muy baja, tanto en el recuento de totales y de viables. El mismo autor concluye que sus resultados generan dudas sobre la concentración real de estos parásitos en el agua, ya que los índices de prevalencia en la población son mucho mayores. De igual manera, Campos (7), reportó la presencia de huevos de helminto en aguas residuales de $0.96 \mathrm{~h} /$ litro, pero no realizó estudios de viabilidad. Los resultados obtenidos en este estudio son concordantes por los reportados por Valbuena en Venezuela (5).

Una situación en particular agrava la problemática, ya que la disposición de estos lodos se realiza sin seguir las recomendaciones internacionales que establecen que no pueden ser dejados al descubierto en época de invierno, deben estar lejos de aguas superficiales y/o subterráneas, de cultivos y no pueden estar en contacto directo con personas y animales, estas recomendaciones no se cumplen en el lugar donde se realizó este estudio.

En cuanto a los resultados obtenidos en lodos, son comparables por lo reportado por Campos (17), en ambos casos, valores superiores a los recomendados como seguros por la Norma Oficial Mexicana. Guzmán 
\& Campos (6), reportaron, pero esta vez utilizando el protocolo de la Agencia de Protección Ambiental de los Estados Unidos (EPA), valores muy superiores, en donde Áscaris sp fue el parásito mas prevalente y su viabilidad fue $<1$ huevo viable. En estudios similares, Lozada (18), Maya (19) y Jiménez (20), demostraron la presencia de una alta concentración de huevos de helmintos, que representan riesgo para la salud pública.

Actualmente, la normatividad establecida en Colombia con respecto a los usos del agua y residuos líquidos, está contemplada en el Decreto 1594 de 1984 (21), donde no se encuentra establecida ninguna pauta con respecto a la calidad parasitológica de las aguas residuales que serán vertidas a las fuentes de agua o que serán utilizadas para el riego de cultivos. Sin embargo, y gracias a los pocos trabajos que han abordado esta problemática, las entidades nacionales encargadas de establecer las normas están elaborando el decreto que incluirá la cuantificación de huevos de helminto y su uso dependiendo de la calidad parasitológica de las aguas residuales y sus subproductos. Se espera que para el año 2013 dicho Decreto sea puesto en práctica.

El presente trabajo confirma la presencia de huevos de helmintos viables tanto en las aguas residuales como en los lodos, en valores superiores a 1 huevo de helminto viable/L de agua y mayor a 1 en $2 \mathrm{~g} / \mathrm{ST}$ en muestras de lodos o biosólidos, lo que se considera como riesgo potencial para la salud pública por la OMS y alerta sobre la posible presencia de estos huevos de helmintos en los alimentos que han sido regados con estas aguas o que han estado en contacto con los lodos provenientes de esta planta de tratamiento. Esta situación sugiere la urgente intervención de los entes reguladores para controlar el vertimiento de estas aguas a las fuentes receptoras y controlar el uso de estas aguas para el riego de cultivos.

La OMS y Norma Oficial Mexicana, han establecido una serie de pautas, con respecto a la posible utilización de las aguas residuales tratadas y los lodos pos-tratamiento con fines agrícolas siempre y cuando se garantice que el tratamiento reduzca las cargas contaminantes a niveles considerados como seguros (1 huevo de helminto viable), para los humanos y los animales. En ninguno de los casos estas pautas se cumplen, por lo que se descarta el contacto directo de estas aguas y lodos con personas. Por lo tanto se recomienda su uso para rellenos sanitarios o reforestación en zonas alejadas de poblaciones humanas y animales y fuentes de agua. 
Se pudo observar, que aunque se ve una reducción considerable entre la cantidad de huevos que ingresan a la planta de tratamiento con el agua cruda y salen en el agua tratada, la planta de tratamiento, con respecto a la cantidad de huevos de helmintos que está vertiendo al emisario final, no se encuentra dentro de la normatividad establecida por la OMS. Este dato es totalmente relevante, ya que al presentarse una cantidad de huevos de helmintos mayor a lo establecido por esta norma se concluye que existe un riesgo para la salud de las personas que estén en contacto directo o indirecto con estas aguas y que la planta de tratamiento de aguas residuales del municipio el Rosal no posee un sistema eficiente de remoción de huevos de helminto

Agradecimientos: A la Facultad de Medicina y la Facultad de Ciencias de la Universidad Nacional de Colombia. A la Corporación Autónoma Regional por el apoyo logístico, especialmente al Ingeniero Martin Mazo por su colaboración.

\section{REFERENCIAS}

1. World Health Organization. Weekly epidemiological record. 2006; 16:145-464.

2. World Health Organization. Integrated guide to Sanitary Parasitology. Regional Office for eastern Mediterranean. Regional centre for environmental health activities. 120. Amman-Jordan. 2004.

3. Cáceres E, Castaño De Romero L, Estupiñán D, López MC, Páez S, Pinilla CA, Santacruz MM. En: Corredor A, Arciniegas E, Hernández CA eds. Parasitismo intestinal. Ministerio de Salud, Instituto Nacional de Salud. Bogotá: Instituto Nacional de Salud. 2000; 67-8.

4. Fernández J, Reyes P, Moncada L, López M, Chaves MP, Knudson A, Ariza Y. Tendencia y prevalencia de las geohelmintiasis en La Virgen, Colombia 1995-2005. Rev. Salud Pública (Bogotá). 2007; 9:289-296.

5. Valbuena D, Díaz O, Botero L y Cheng R. Detección de helmintos intestinales y bacterias indicadoras de contaminación en aguas residuales tratadas y no tratadas. Interciencia. 2002; 27: 710-714.

6. Guzmán C y Campos C. Indicadores de contaminación fecal en biosólidos aplicados en agricultura. Universitas Scientarium. 2004; 9:59-67.

7. Campos C, Cárdenas M, y Guerrero A. Comportamiento de los diferentes indicadores de contaminación fecal en diferente tipo de aguas de la sabana de Bogotá (Colombia). Universitas Scientarium. 2008; 13:103-108.

8. Corporación Autónoma Regional de Cundinamarca. Manual de operación y mantenimiento de la planta de tratamiento de aguas residuales domésticas de $\mathrm{El}$ Rosal - Cundinamarca. Consorcio Essere Ltda. Gelver Ayala. Contrato de obra 19705. Operación y mantenimiento de las plantas de tratamiento de aguas residuales y sus estaciones de bombeo ubicadas en los municipios de los sectores I y II de la cuenca alta del río Bogotá; 2006.

9. Ayres MR, Mara DD. Analysis of Wastewater for Use in Agriculture-A Laboratory Manual of Parasitological and bacteriological Techniques. World Health Organization; 1996. pp. 1-35. 
10. Secretaría de Medio Ambiente y Recursos Naturales. Estados Unidos Mexicanos. Norma Oficial Mexicana NOM-004-SEMANART-2002, Protección Ambiental Lodos y Biosólidos, especificaciones y límites máximos permisibles de contaminantes para su aprovechamiento y disposición final; 2003.

11. Victórica J, Galván M. Preliminary testing of a rapid coupled methodology for quantitation/ viability determination of helminths eggs in raw and treated wastewater. Wat Res. 2003, 37:1278-1287.

12. Salas AM. Centro Panamericano de Ingeniería Sanitaria y Ciencias del Ambiente (CEPIS). Inventario de la situación actual de las aguas residuales domésticas en Colombia. Sistemas integrados de tratamiento y uso de aguas residuales en América Latina: realidad y potencial. Lima, Perú; 2003.

13. Silva J, Torres P, Madera C. Reuso de águas residuales domésticas em agricultura. Una revisión. Agron Col. 2008; 26(2):347-359.

14. Maya C, Jiménez BE, Barrios JA. Evaluación microbiológica de un lodo residual en México: Perspectivas de reuso. XXVIII Congreso Interamericano de Ingeniería Sanitaria y Ambiental. Cancún, México; 27 al 31 de octubre de 2002.

15. Jimenez B, Maya C, Galván M. Helminth ova control in wastewater and sludge for advanced and conventional sanitation. Wat. Scie Tech. 2007, 56(5):43-51.

16. Campos C, Beltrán M. Evaluación de la concentración y viabilidad de huevos de helmintos en matrices sólidas relacionadas con la disposición de biosólidos para uso agrícola. Memorias XIV Congreso Colombiano de Parasitología y Medicina Tropical. X Simposio PECET. Revista Biomédica. 2009; 29. Suppl (1):46-47.

17. Campos C, Beltrán M. Determinación de huevos de helminto, Giardia y Cryptosporidium como herramienta para evaluar el riesgo sanitario en aguas potables y residuales. Memorias XIV Congreso Colombiano de Parasitología y Medicina Tropical. X Simposio PECET. Biomédica. 2009, 29. Suppl.(1):44-46.

18. Lozada P, Escobar JC, Pérez A, Imery R, Nates P, Sánchez G, Sánchez M, Bermúdez A. Influencia del material de enmienda en el compostaje de lodos de Plantas de tratamiento de aguas residuales-PTAR. Revista ingeniería e Investigación. 2005; 25: 53-61.

19. Maya C, Jiménez B, Schwartzbrod J. Comparison of techniques for the detection of Helminth ova in Drinking water and wastewater. Water environment research. 2006; 78:118-124.

20. Jiménez B. Helminth ova removal from wastewater for agriculture and aquaculture reuse. Wat Sci Tech. 2007;55:485-493.

21. Republica de Colombia. Decreto 1594 de 1984: Usos del agua y residuos líquidos. (Internet). Disponible en: http://www.alcaldiabogota.gov.co/sisjur/normas/Norma1. jsp?i=18617. Consultado: Julio de 2012. 